\title{
Epithelial apical glycosylation changes associated with thin endometrium in women with infertility - a pilot observational study
}

Marina M. Ziganshina ${ }^{1 *} \mathbb{D}$, Nataliya V. Dolgushina ${ }^{2,3} \mathbb{D}$, Galina V. Kulikova ${ }^{4} \mathbb{D}$, Nafisa M. Fayzullina ${ }^{4} \mathbb{D}$, Ekaterina L. Yarotskaya ${ }^{5}$ (D), Nailia R. Khasbiullina ${ }^{1} \mathbb{D}$, Nigora F. Abdurakhmanova ${ }^{6}$, Aleksandra V. Asaturova ${ }^{4} \mathbb{D}_{\text {, }}$ Alexander I. Shchegolev ${ }^{4}$ (D), Alina A. Dovgan ${ }^{6}$ (D) and Gennady T. Sukhikh ${ }^{1,3}$ (D)

\begin{abstract}
Background: Low endometrial receptivity is one of the major factors affecting successful implantation in assisted reproductive technologies (ART). Infertile patients with thin endometrium have a significantly lower cumulative clinical pregnancy rate than patients with normal endometrium. Molecular pathophysiology of low receptivity of thin endometrium remains understudied. We have investigated composition of glycocalyx of the apical surface of luminal and glandular epithelial cells in thin endometrium of infertile women.

Methods: Thirty-two patients with tubal-peritoneal infertility undergoing in vitro fertilization (IVF) were included in the study. Endometrial samples were obtained in a natural menstrual cycle. Patients were divided into two groups: patients with normal endometrium $(\geq 8 \mathrm{~mm})$ and with thin endometrium $(<8 \mathrm{~mm})$. Histochemical and immunohistochemical analysis of paraffin-embedded endometrial samples was performed using six biotinylated lectins (UEA-I, MAL-II, SNA, WL, ECL, Con A) and anti-Le ${ }^{Y}$ and MECA-79 monoclonal antibodies (MAbs).
\end{abstract}

Results: Complex glycans analysis taking into account the adjusted specificity of glycan-binding MAbs revealed 1.3 times less expression of MECA-79 glycans on the apical surface of the luminal epithelial cells of thin endometrium compared to normal endometrium; this deficiency may adversely affect implantation, since MECA-79 glycans are a ligand of L-selectin and mediate intercellular interactions. The glycans containing a type-2 unit Galß1-4GlcNAc $\beta$ (LacNAc) but lacking sulfo-residues at 6-OH of GlcNAc $\beta$, and binding to MECA-79 MAbs were found; they can be considered as potential markers of endometrium receptivity.

Expression of the lectins-stained glycans on the apical surfaces of the luminal and glandular epithelial cells did not differ significantly. Correlation between the expression of difucosylated oligosaccharide Le $e^{Y}$ on the apical surfaces of the luminal and glandular epithelial cells was found in patients with thin endometrium and recurrent implantation (Continued on next page)

\footnotetext{
* Correspondence: mmz@mail.ru

'Laboratory of Clinical Immunology, National Medical Research Center for Obstetrics, Gynecology, and Perinatology named after Academician V.I.

Kulakov of the Ministry of Healthcare of Russian Federation, Oparina str. 4, Moscow, Russian Federation 117997

Full list of author information is available at the end of the article
}

C C The Author(s). 2021 Open Access This article is licensed under a Creative Commons Attribution 4.0 International License, which permits use, sharing, adaptation, distribution and reproduction in any medium or format, as long as you give appropriate credit to the original author(s) and the source, provide a link to the Creative Commons licence, and indicate if changes were made. The images or other third party material in this article are included in the article's Creative Commons licence, unless indicated otherwise in a credit line to the material. If material is not included in the article's Creative Commons licence and your intended use is not permitted by statutory regulation or exceeds the permitted use, you will need to obtain permission directly from the copyright holder. To view a copy of this licence, visit http://creativecommons.org/licenses/by/4.0/ The Creative Commons Public Domain Dedication waiver (http://creativecommons.org/publicdomain/zero/1.0/) applies to the data made available in this article, unless otherwise stated in a credit line to the data. 
(Continued from previous page)

failure. A similar relationship was shown for mannose-rich glycans.

Conclusions: Specific features of key glycans expression in epithelial compartments of thin endometrium may be essential for morphogenesis of the endometrial functional layer and explain its low receptivity.

Keywords: Thin endometrium, Infertility, Assisted reproductive technologies (ART), Lectin histochemistry, Glycans, Glycocalyx, MECA-79

\section{Background}

Endometrial thickness was proposed to be a prognostic criterion for pregnancy by Gonen et al. in 1989 [1]. Since then, the concept of minimal valid endometrial thickness was actively used in clinical practice. Endometrium less than 6-8 $\mathrm{mm}$ is now considered to be "thin" [2-4]. A predictive value of this indicator is contradictory, since pregnancies have been reported to occur in patients with endometrium 4-5 $\mathrm{mm}$, suggesting that receptivity might be not necessarily related to endometrial thickness [5, 6]. However, many experts agree that insufficient endometrial thickness may reduce the cumulative clinical pregnancy rate, live birth rate [7], and be associated with small for gestational age neonates and obstetric complications caused by poor placentation [8].

Current data on molecular changes in thin endometrium, reflecting pathophysiological mechanisms of endometrial receptivity reduction [9-11] are very limited. Endometrial receptivity can be only assessed in nonconception cycles, where endometrial thickness and receptivity may vary. Therefore, a complex assessment of the molecular composition of the main functional structures of endometrium in a natural cycle is needed. This could allow prediction of the endometrium potential for blastocyst acceptance and trophoblast invasion. Molecular research should be obviously focused on the key molecules involved in intercellular interactions mediating these processes.

Human endometrium, where most proteins on the cellular surface are glycosylated, demonstrate particularly rich in glycans expression [12, 13]. Glycoconjugates are associated with the apical surfaces of epithelial cells, which contact with blastocyst during implantation. Secretions of the endometrial glands contain a lot of glycoproteins and mucins that promote specific uterine functions [14, 15]. However, even though the studies of glycans associated with the apical surface of luminal and glandular epithelial cells have been performed earlier [15-19], there is no data on the composition of glycocalyx of thin endometrium.

The analysis of functional carbohydrate residues, which are important part of glycoproteins, glycolipids, and extracellular matrices of endometrial tissue, may reveal the carbohydrate phenotype (the "glycocode") of thin endometrium. Detection of the coherent changes of glycans in the endometrial epithelial structures may determine their importance for normal and thin endometrium and their impact on receptivity. In this regard, the study was aimed to evaluate complex changes in the composition of glycocalyx and to analyze the expression of glycans in luminal and glandular epithelial cells of normal and thin endometrium in patients with infertility.

\section{Methods}

Patients and endometrial biopsy samples

Thirty-two patients with tubal-peritoneal infertility undergoing IVF treatment were included in the study. Endometrial samples were obtained in a natural menstrual cycle around day 6 after ovulation (cycle day 20-22, «implantation window»). The patients did not receive any hormonal therapy for at least 2 months before the procedure. All samples were examined by the same pathologist using the dating criteria described by Noyes RW [20].

In our previous study [21] of 154 infertile patients stratified into 2 groups (43 patients with endometrial thickness $9.3 \pm 0.9 \mathrm{~mm}$ who achieved pregnancy by IVF, and 110 patients with endometrial thickness $7.2 \pm 0.9$ $\mathrm{mm}$, who failed to conceive $(p<0.0001))$, we have found that a threshold of endometrial thickness, with which the odds of pregnancy and the validity of the logistic regression model increased, was $8.0 \mathrm{~mm}$ (AUC $=86.7 \%$, Se $=97.7 \%, \mathrm{Sp}=75.7 \%)$. Basing on this observation, for the current study we have set the threshold of endometrial thickness $8 \mathrm{~mm}$.

Depending on the endometrium thickness, the patients were divided into two groups: patients with "thin" endometrium ( $<8 \mathrm{~mm}, n=14)$, group 1 , and patients with normal endometrium ( $\geq 8 \mathrm{~mm}, n=18$ ), group 2 .

Inclusion criteria were normal karyotypes of the patient and her partner; age 18-40 years; body mass index (BMI) $18-29.9 \mathrm{~kg} / \mathrm{m}^{2}$. Exclusion criteria were: contraindications to IVF; pathozoospermia in the partner; use of donor gametes or surrogacy; poor ovarian response to stimulation; absence of good quality blastocysts; and IVF complications in the studied cycle.

The IVF program (the next month after endometrial biopsy) involved ovarian stimulation with recombinant follicle-stimulating hormone $(\mathrm{rFSH})$ or a combination of $\mathrm{rFSH}$ and luteinizing hormone $(\mathrm{LH})$ and treatment with 
gonadotropin-releasing hormone antagonists (GnRHant). The ovulation trigger (HCG 8.000-10.000 IU) was administered when leading follicles reached $17 \mathrm{~mm}$ or more in diameter. Transvaginal puncture (TVP) was performed $36 \mathrm{~h}$ after the administration of the ovulation trigger.

Oocytes were fertilized by intracytoplasmic sperm injection (ICSI), because of subfertility of the male partner and low fertilization rate in the previous IVF cycle. Morphological evaluation of the embryos was performed on the 5th day after TVP, according to Gardner et al. classification [22].

The embryo transfer (ET) was performed on the 5th day after TVP in a fresh cycle. One blastocyst of the best quality was transferred into the uterine cavity. Biochemical pregnancy was stated if the level of serum B-HCG increased 14days after ET; a clinical pregnancy was stated if ultrasound scan showed the gestational sac 21 days after ET.

\section{Antibodies}

Purified mouse anti-blood group Lewis ${ }^{\mathrm{Y}}\left(\mathrm{Le}^{\mathrm{Y}}\right)$ monoclonal antibodies, clone LWY/1463, Abcam, ab 219,336, 1/ 200 (anti-Le ${ }^{\mathrm{Y}} \mathrm{MAbs}$ ) and purified rat anti-mouse peripheral lymph node addressin (PNAd) carbohydrate epitope monoclonal antibodies, clone MECA-79, BD Pharmingen, Cat. No 553863, 1/100 (MECA-79 MAbs) were used to determine the expression of the glycans epitope in the endometrium. The anti-Le ${ }^{\mathrm{Y}}$ MAbs recognize a difucosylated oligosaccharide $\mathrm{Le}^{\mathrm{Y}}$ (major specificity Fuc $\alpha 1-2$ Gal $\beta 1-4$ (Fuc $\alpha 1-3)$ GlcNAc $\beta$-R). MECA-79 MAbs react with carbohydrate epitopes which are a high-affinity L-selectin ligand (CD34, GlyCAM-1, MAdCAM-1). As previously reported, MECA-79 epitope contain (6-O$\mathrm{Su})$ GlcNAc $\beta$-residue [23].

\section{Lectins}

A panel of six selected biotinylated lectins (Vector Labs, USA) was used to study the glycocalyx composition in the structural elements of the endometrium: MAL-II (Maackia amurensis lectin-II, binding specificity NeuNAca2-3Galß); SNA (Sambucus nigra lectin, binding specificity NeuNAca2-6Gal/GalNAc); ECL (Erythrina cristagalli lectin, binding specificity Gal $\beta 1$ 4GlcNAc $\beta$ (LacNAc, type-2 unit)); UEA-I (Ulex europaeus agglutinin-I, binding specificity $\mathrm{H}$ type 2 antigen L-Fuc $\alpha 1-2$ Gal $\beta 1-4$ GlcNAc $\beta 1$ and difucosylated oligosaccharide $\mathrm{Le}^{\mathrm{Y}}$ ); VVL (Vicia villosa lectin, binding specificity GalNAco1-Ser/Thr and Galo1-3GalNAc); Con A (Concanavalin A from Canavalia ensiformis, binding specificity D-Glco and D-Man $\alpha$ (terminal or 1-2-linked) in high mannose, intermediate and small complex $\mathrm{N}$ linked sequences) [24]. An optimal concentration for each lectin from the panel, which allowed the maximum labeling with minimum non-specified background, was $10 \mu \mathrm{g} / \mathrm{ml}$ for MAL-II and VVL, $5 \mu \mathrm{g} / \mathrm{ml}$ for UEA-1, and $2,5 \mu \mathrm{g} / \mathrm{ml}$ for lectins SNA, ECA, and Con A.

\section{Lectin histochemistry and immunohistochemistry}

For lectin histochemistry, the paraffin sections, after dewaxing and hydration, were treated with $3 \%$ hydrogen peroxide for $10 \mathrm{~min}$ to inhibit the endogenous peroxidase, rinsed with distilled water, and treated with an avidin-biotin kit (Vector Labs, USA) to block endogenous avidin-binding activity. The sections were rinsed with phosphate-buffered saline (PBS) and incubated with Carbo-Free block solution (Vector Labs, USA) for 30 min to reduce the background staining. Then the sections were incubated for $30 \mathrm{~min}$ at room temperature with biotinylated lectins solution in PBS. After washing the sections were incubated with the avidin-biotinperoxidase complex (ABC, Vector Labs, USA) for 30 min. After 3 washes in PBS, the peroxidase activity sites were visualized using DAB solution.

For immunohistochemistry, endometrial samples were fixed in $10 \%$ buffered formaldehyde for $24 \mathrm{~h}$, embedded in paraffin, and $3 \mu \mathrm{m}$ thick sections were prepared for immunohistochemical (IHC) investigation. IHC staining of citrate-pretreated sections (90 for $20 \mathrm{~min}$ ) was performed using MAbs: anti-Le ${ }^{Y}$ MAbs $(1 \mu \mathrm{g} / \mathrm{ml})$ and MECA-79 MAbs $(5 \mu \mathrm{g} / \mathrm{ml})$. The binding of Le ${ }^{\mathrm{Y}}$ was detected by Dako REAL ${ }^{\mathrm{Tm}}$ EnVision $^{\mathrm{Tm}}$ Detection System (Dako, Denmark). The binding of MECA-79 MAbs was revealed with the use of a goat anti-rat $\operatorname{IgG}(\mathrm{H}+\mathrm{L})$ $(1 \mu \mathrm{g} / \mathrm{ml}$, Vector Laboratories, USA), conjugated with peroxidase. Slides were counterstained with hematoxylin, dehydrated, cleared, and mounted in Shandon-mount to be examined under light microscopy.

\section{Controls for lectin and MAbs staining}

Negative controls were carried out by replacement of the lectins and MAbs solution with a buffer (PBS, pH 7.4) to identify non-specific binding of avidin-peroxidase to the tissue or residual endogenous peroxidase activity. An irrelevant rat IgM antibody (purified rat IgM, $\mathrm{K}$ isotype control clone R4-22, BD Pharmingen, Cat. No 553940), and mouse IgG1 ( $\kappa$ isotype control 15-6E10A7, Abcam, ab 170190) MAbs were used instead the primary antibody. Sections were also incubated with lectins in the presence of an appropriate competing sugar: $0.2 \mathrm{M}$ $\alpha$-methyl mannoside for Con A, $0.2 \mathrm{ML}$-fucose for UEA-1, 0.2 M galactose for ECL, $0.2 \mathrm{M} \mathrm{N}$-acetylgalactosamine for VVL. The lung carcinoma, breast cancer lymph nodes and placental terminal villi were used as positive controls for staining of endometrium with an anti-Le ${ }^{\mathrm{Y}}$ antibody, MECA-79 antibody and with lectins ConA and SNA, respectively. 


\section{Neuraminidase digestion}

For lectins ECL, SNA, and MAL-II, sialic acid was removed by pretreating the sections with $50 \mathrm{mU} / \mathrm{ml}$ neuraminidase (N) from Vibrio cholerae (Type III, SigmaAldrich, USA). Neuraminidase pre-treatment was used for $2 \mathrm{~h}$ at $37^{\circ} \mathrm{C}$, as described by Jones CJP [25].

\section{Assessment of staining location and reactivity intensity}

A grid, $1 \mathrm{~mm} \times 1 \mathrm{~mm}$, divided into 100 fields, was used for staining assessment. The reaction intensity was assessed by two independent researchers "blindly" (the samples were encrypted). In each examination 10 randomly selected equidistant fields of view were studied using a random number generator. The measurements were processed automatically as average values of optical density on the apical surface of luminal epithelial cells and glandular epithelial cells using image analysis with NIS Elements Advanced Research 3.2 program (Laboratory Imaging LTD, Czech Republic).

\section{Microarray chip analysis}

The microarray applied in the study was a polymer-coated glass slide with $\mathrm{N}$-hydroxysuccinimide-derivatized surface, produced by Schott-Nexterion (Germany), with 651 spacered glycans in $50 \mu \mathrm{M}$ solutions at 6 replicates [26]. A complete list of printed glycans is presented in an additional file (Table S1). A sample of anti- $\mathrm{Le}^{\mathrm{Y}}$ MAbs or MECA-79 MAbs (both at concentration $5 \mu \mathrm{g} / \mathrm{mL}$ ), diluted in PBS (Sigma Aldrich, USA) containing $0.1 \%(\mathrm{v} / \mathrm{v})$ of Tween 20 (Merck, USA) and 1\% BSA (Sigma, USA), was placed onto the chip surface and incubated in a humidified dark chamber at $37 \mathrm{C}$ and mild rotation (32-34 rot/ $\mathrm{min}$ ) for $1 \mathrm{~h}$. Then the chip was washed with PBS containing $0.05 \%$ Tween 20 (PBS-0.05\%) and incubated with biotinylated goat-anti-mouse antibodies (Thermo Fisher Scientific, USA) at concentration $10 \mu \mathrm{g} / \mathrm{ml}$ in PBS- $0.1 \%$ under the same conditions. Then the chip was washed with PBS- $0.05 \%$ and incubated with streptavidin-Alexa555 conjugate (Thermo Fisher Scientific, USA) at $1 \mu \mathrm{g} / \mathrm{ml}$ in PBS- $0.1 \%$ at room temperature $\left(20^{\circ} \mathrm{C}\right)$ for $45 \mathrm{~min}$ and mild rotation. The chip was air-dried and scanned by InnoScan $710 \mathrm{AL}$ (Innopsys, France) at $\lambda_{\mathrm{ex}} 543 \mathrm{~nm}$ and $10 \mu \mathrm{m}$ resolution. The obtained data were processed using ScanArray Express 4.0 software, a fixed $80 \mu \mathrm{m}$-diameter circle method and Microsoft Excel software. Data for each glycan on the array are reported as median relative fluorescence units (RFU) of 6 replicates. The median deviation was measured as an interquartile range. A signal with fluorescence intensity 5 times exceeding the background value was considered significant.

\section{Statistical analysis}

A statistical software package Statistica 10 (USA) was used to perform a $\chi^{2}$ test to compare categorical variables, t-test, linear regression, or Mann-Whitney $U$ test. The odds ratio (OR) adjusted for confounders and calculated using logistic regression was used as a measure of association to compare binary data. Correlation analysis was performed with the use of Spearman's rank correlation coefficient. Differences between statistical values were considered statistically significant at a confidence level $p<0.05$.

\section{Results \\ Clinical results}

The average age and BMI of patients in the studied groups did not differ significantly $(p>0.05)$. There were also no differences in menstrual function, gravidity, parity, hormonal tests, and somatic morbidity. Patients with thin endometrium often had a history of endometrial polyps. There were no differences in cumulative doses of gonadotropins (GT), duration of ovarian stimulation, or in drugs used for ovarian stimulation (Table 1). A larger number of excellent blastocysts were obtained from patients with normal endometrial thickness (Table 2).

The pregnancy rate in patients with endometrial thickness $\geq 8 \mathrm{~mm}$ was $66.7 \%$ (12 out of 18 women), while patients with thin endometrium failed to become pregnant ( 0 out of 14) $(p=0.0001)$. In the multivariate analysis with logistic regression, taking into account the number of excellent blastocysts, the OR of pregnancy, depending on the endometrium thickness, was $1.47(95 \% \mathrm{CI}=1.05 ; 2.5)$.

\section{Intragroup comparison and correlation analysis of the glycans expression levels on the apical surface of the luminal and glandular epithelial cells in women with normal and thin endometrium}

The glycans expression on the apical surfaces of luminal and glandular epithelial cells was assessed, and correlations between the expression levels were calculated for each studied group separately. The data obtained for each group were analyzed thereafter to find out the patterns of the glycans expression in normal and thin endometrium. In both groups, the glycans expression patterns were similar in both endometrial structures, as confirmed by correlation and comparative analysis with linear regression (Tables 3 and 4). In case of thin endometrium, a moderate positive correlation was observed for the contents of glycoconjugates stained with anti-Le ${ }^{\mathrm{Y}}$ MAbs (an example is one of the photomicrographs of Fig. 1a) and Con A (Fig. 1b), UEA-I, N + ECL on the apical surface of the luminal epithelial cells, and the contents of the same glycoconjugates on the apical surface of glandular cells (Table 3). In patients with normal endometrium, a moderate positive correlation was found for staining with lectins UEA-I and N + ECL in the two endometrial structures (Table 4). It should be noted that despite the revealed significant relationship between the 
Table 1 Selected characteristics of the patients, included in the study

\begin{tabular}{|c|c|c|c|}
\hline Features & Group $1(n=14)$ & Group $2(n=18)$ & $p$-value \\
\hline Age, years ${ }^{b}$ & $33.0 \pm 5.5$ & $33.3 \pm 3.3$ & 0.8611 \\
\hline $\mathrm{BMl}, \mathrm{kg} / \mathrm{m}^{2 \mathrm{~b}}$ & $22.4 \pm 3.0$ & $22.5 \pm 2.6$ & 0.9267 \\
\hline Endometrial polyps ${ }^{\text {a }}$ & $5(35.7 \%)$ & $2(11.1 \%)$ & 0.0948 \\
\hline Gravidity $^{c}$ & $0(0-1)$ & $1(0-2)$ & 0.2706 \\
\hline Parity $^{c}$ & $0(0-0)$ & $0(0-0)$ & 0.6622 \\
\hline Number of IVF cycles ${ }^{c}$ & $0(0-2)$ & $1(0-2)$ & 0.4250 \\
\hline $\mathrm{FSH}, \mathrm{mlU} / \mathrm{mL}^{\mathrm{b}}$ & $7.4 \pm 2.8$ & $7.8 \pm 2.2$ & 0.5781 \\
\hline $\mathrm{AMH}, \mathrm{ng} / \mathrm{mL}^{\mathrm{b}}$ & $3.5 \pm 3.0$ & $4.1 \pm 3.9$ & 0.6377 \\
\hline Stimulation duration, days ${ }^{b}$ & $8.9 \pm 0.7$ & $9.2 \pm 1.3$ & 0.5432 \\
\hline GT cumulative dose, IU & $1419.6 \pm 371.6$ & $1612.5 \pm 532.3$ & 0.2581 \\
\hline
\end{tabular}

$B M I$ body mass index, FSH follicle-stimulating hormone, $A M H$ anti-Mullerian hormone, GT gonadotropins

${ }^{a}$ Data are presented as absolute numbers and $\%, x^{2}$ test

${ }^{\mathrm{b}}$ Data are presented as mean \pm standard deviation, $\mathrm{t}$-test

c Data are presented as medians (with interquartile range), Kruskal-Wallis test

expression of subterminal residues Gal $\beta 1-4$ GlcNAc $\beta 1$ $(\mathrm{N}+\mathrm{ECL})$ in the studied endometrial structures of patients of both groups, their contents alterations were reciprocal, in contrast to the above pattern. The comparative results of lectins staining in the glycocalyx of luminal and glandular epithelial cells of endometrium in the studied groups are shown in Tables 3 and 4.

\section{Intergroup comparison of the glycans expression on the} apical surface of the luminal and glandular epithelial cells in women with normal and thin endometrium

An intergroup comparison revealed a decreased expression of carbohydrate epitopes MECA-79 on the apical surface of the epithelial cells of thin endometrium, compared to normal ( $p=0.033)$ (Fig. 2). Differences in glycoconjugates expression on the apical surface of the glandular cells were not found. An additional file shows this in more detail (see Table S2).

\section{Carbohydrate specificity of anti-Le ${ }^{\mathrm{Y}}$ and MECA-79 MAbs}

Microarray chip analysis of MAbs showed that anti-Le ${ }^{Y}$ MAbs exclusively bound to difucosylated oligosaccharide
Le $^{\mathrm{Y}}$ (Fig. 3 a). The glycans, most actively binding to MECA-79 MAbs were those containing a type-2 unit LacNAc (Gal $\beta 1-4 G l c N A c \beta)$, and especially an oligolactosamine derivative Gal $\beta 1-4$ GlcNAc $\beta 1-3($ GlcNAc $\beta 1-6)$ Gal $\beta 1-4 G l c N A c \beta$. Lower but still significant binding was detected between MECA-79 MAbs and a $\mathrm{Le}^{\mathrm{Y}}$ derivative GalNAc $\beta 1-3$ (Fuc $\alpha 1-2)$ Gal $\beta 1-4$ (Fuc $\alpha 1-3)$ GlcNAc $\beta$; $\quad$ a fucose-rich sulfated SialylLe ${ }^{\mathrm{X}}$ residue Neu5Aco23Gal $\beta 1-4(2-\mathrm{O}-\mathrm{Su}-\mathrm{Fuc} \alpha 1-3)$ GlcNAc $\beta$; and an $\alpha-$ lactosamine substituted derivative 6-Bn-Gal $\alpha 1-4(6-\mathrm{Bn})$ GlcNAc $\beta$ (where $\mathrm{Bn}$ is a benzyl residue) (Fig. 3b). Moreover, the 2nd and the 4th glycans from this list were not detected in humans before. I.e. MECA-79 MAbs specifically bind to LacNAc-containing oligosaccharides, but do not bind to mucin core 1 glycans of the microarray.

\section{Discussion}

In this study, we explored the concept that the carbohydrate phenotype of endometrial structures may reflect a decreased receptivity of thin endometrium. We focused on glycans, basing on: 1) hormone-dependent change of the glycosylation pattern in the menstrual cycle $[16,27$,

Table 2 Characteristics of gametes and embryos of the patients included in the study

\begin{tabular}{|c|c|c|c|}
\hline Feature & Group $1(n=14)$ & Group $2(n=18)$ & p-level \\
\hline Normal sperm $^{a}$ & $8(57.1 \%)$ & $14(77.7 \%)$ & 0.3193 \\
\hline Average number of mature oocytes per patient ${ }^{b}$ & $6(3-7)$ & $4(4-7)$ & 0.7612 \\
\hline Average number of zygotes per patient ${ }^{b}$ & $6(2-7)$ & $4(3-6)$ & 0.6622 \\
\hline Average fertilization rate ${ }^{c}$ & $0.72 \pm 0.15$ & $0.75 \pm 0.23$ & 0.7648 \\
\hline Average number of blastocyst per patient ${ }^{\mathrm{b}}$ & $4(1-5)$ & $3(2-4)$ & 0.9848 \\
\hline Average level of blastulation ${ }^{c}$ & $0.68 \pm 0.26$ & $0.77 \pm 0.26$ & 0.3143 \\
\hline Average number of excellent blastocysts per patient ${ }^{b}$ & $0(0-2)$ & $1.5(1-3)$ & 0.0303 \\
\hline
\end{tabular}

${ }^{a}$ Data are presented as absolute numbers and \%, $\mathrm{x} 2$ test

${ }^{\mathrm{b}}$ Data are presented as medians (with interquartile range), Mann-Whitney $\mathrm{U}$ test:

${ }^{c}$ Data are presented as mean \pm standard deviation, $t$-test 
Table 3 Glycoconjugates contents on the apical surface of the luminal and glandular epithelial cells in thin endometrium

\begin{tabular}{lllll}
\hline Lectins and MAbs & Luminal cells & Glandular cells & $\mathbf{r}_{\mathbf{s}}$ & $\boldsymbol{p}$-value \\
\hline MAL II & $0.29 \pm 0.07(0.18-0.41)$ & $0.27 \pm 0.08(0.15-0.45)$ & 0.3475 & 0.1717 \\
N + MAL I & $0.16 \pm 0.04(0.09-0.24)$ & $0.10 \pm 0.03(0.05-0.17)$ & 0.4510 & 0.0692 \\
UEA I & $0.24 \pm 0.11(0.06-0.50)$ & $0.17 \pm 0.10(0.08-0.41)$ & 0.6472 & 0.0036 \\
SNA & $0.34 \pm 0.09(0.19-0.48)$ & $0.22 \pm 0.08(0.10-0.38)$ & 0.2675 & 0.2832 \\
N+SNA & $0.32 \pm 0.09(0.14-0.44)$ & $0.17 \pm 0.07(0.07-0.29)$ & 0.5697 & 0.0135 \\
ECL & $0.35 \pm 0.12(0.11-0.51)$ & $0.26 \pm 0.10(0.10-0.42)$ & 0.0734 & 0.7720 \\
N+ECL & $0.51 \pm 0.11(0.24-0.68)$ & $0.57 \pm 0.28(0.27-1.49)$ & 0.3773 & 0.0103 \\
WL & $0.25 \pm 0.10(0.15-0.46)$ & $0.24 \pm 0.13(0.07-0.53)$ & 0.1229 \\
Con A & $0.39 \pm 0.09(0.26-0.53)$ & $0.28 \pm 0.06(0.17-0.44)$ & 0.5064 & $\mathbf{0 . 0 3 1 9}$ \\
MECA-79 & $0.33 \pm 0.07(0.25-0.48)$ & $0.19 \pm 0.07(0.03-0.29)$ & 0.1019 & 0.7072 \\
Anti- Le & $0.25 \pm 0.06(0.15-0.39)$ & $0.14 \pm 0.06(0.08-0.34)$ & 0.04791 &
\end{tabular}

Data are presented as mean \pm standard deviation (minimum-maximum)

$r_{s}$ Spearman's rank correlation coefficient between contents of glycoconjugates in luminal and glandular epithelial cells

$P$-value for the Spearman's rank statistic

Italics Bold statistically significant values

$28]$; 2) a specific endometrial glycosylation pattern associated with the blastocyst reception during the implantation window [13, 29-31]; 3) the crucial role of glycans in intercellular adhesion and tissue morphogenesis [32$35]$. We chose to study the epithelial compartments of endometrium in patients with normal and thin endometrium since contact interactions occur on the apical surface of the endometrium epithelial cells, and the glycocalyx of the apical surface of glandular cells reflects their secretory activity and potential for intercellular interactions during trophoblast invasion [36].

Our study was the first to demonstrate that decreased receptivity in infertile female patients is associated with an altered glycan phenotype of thin endometrium. The investigation revealed reduced expression of the L- selectin ligand (MECA-79) in the glycocalyx of epithelial cells in thin endometrium. It should be noted that the glycan composition of MECA-79 epitope was intensively studied before. In particular, it was shown that desialylation of ClyCAM-1 enhanced binding of MECA-79 to ClyCAM-1. In contrast, binding of MECA-79 to ClyCAM-1 and CD34 significantly decreased when the sulfation of these ligands was reduced with chlorate, a metabolic inhibitor of sulfation. Defucosylation also significantly reduced the binding of MECA-79 with ClyCAM-1 [37]. Various authors denoted 6'-sulfated SialylLe $^{\mathrm{X}}$ (Sia $\alpha 2-3(6-\mathrm{O}-\mathrm{Su}) \mathrm{Gal} \beta 1-4(\mathrm{Fuc} \alpha 1-3)$ GlcNAc on core 2 branched O-glycans [38], as well as sulfated extended core 1 mucin-type O-glycans Gal $\beta 1-4(6-\mathrm{O}-$ $\mathrm{Su}$ GlcNAcß1-3GalNAc [23], as the MECA-79 epitope.

Table 4 Glycoconjugates contents on the apical surface of the luminal and glandular epithelial cells in normal endometrium

\begin{tabular}{lllll}
\hline Lectins and MAbs & Luminal cells & Glandular cells & $\mathbf{r}_{\mathbf{s}}$ & $\boldsymbol{p}$-value \\
\hline MAL II & $0.28 \pm 0.11(0.14-0.52)$ & $0.27 \pm 0.08(0.15-0.45)$ & 0.2100 & 0.4711 \\
N + MAL I & $0.15 \pm 0.05(0.10-0.30)$ & $0.12 \pm 0.08(0.04-0.35)$ & 0.0336 & 0.9093 \\
UEA I & $0.24 \pm 0.14(0.07-0.59)$ & $0.22 \pm 0.14(0.05-0.49)$ & 0.5925 & 0.0328 \\
SNA & $0.41 \pm 0.07(0.22-0.51)$ & $0.22 \pm 0.09(0.09-0.41)$ & 0.5040 & 0.0661 \\
N+SNA & $0.34 \pm 0.08(0.21-0.52)$ & $0.19 \pm 0.11(0.06-0.46)$ & 0.1990 & 0.4951 \\
ECL & $0.33 \pm 0.09(0.15-0.52)$ & $0.29 \pm 0.14(0.08-0.62)$ & 0.3812 & 0.1786 \\
N+ECL & $0.57 \pm 0.09(0.39-0.78)$ & $0.55 \pm 0.10(0.39-0.72)$ & 0.5697 & 0.0334 \\
WL & $0.26 \pm 0.09(0.10-0.43)$ & $0.21 \pm 0.09(0.09-0.38)$ & 0.5294 & 0.0628 \\
Con A & $0.35 \pm 0.09(0.15-0.54)$ & $0.30 \pm 0.07(0.17-0.40)$ & 0.1505 & 0.6236 \\
MECA-79 & $0.42 \pm 0.11(0.19-0.65)$ & $0.20 \pm 0.06(0.10-0.31)$ & 0.4476 & -0.2903
\end{tabular}

Data are presented as mean \pm standard deviation (minimum-maximum)

$r_{s}$ Spearman's rank correlation coefficient between contents of glycoconjugates in luminal and glandular epithelial cells $P$ value for the Spearman's rank statistic

Italics Bold statistically significant values 


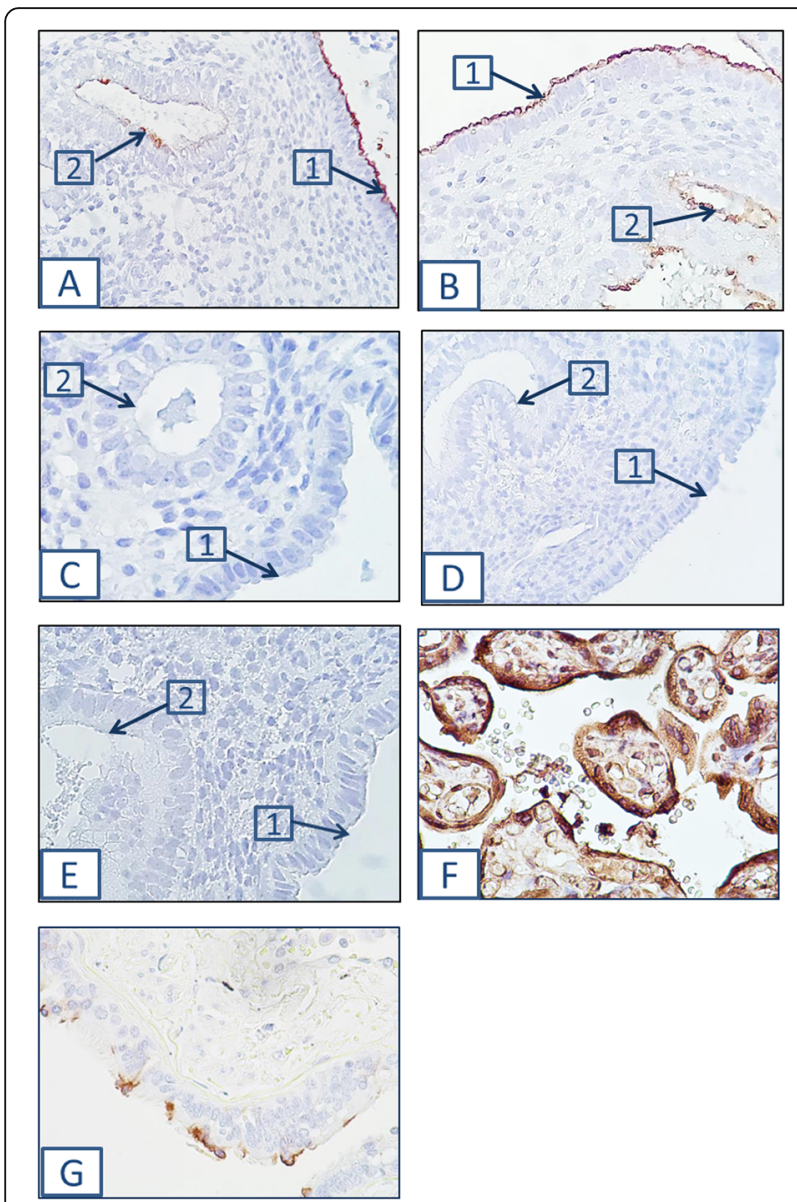

Fig. 1 Glycoconjugates staining in thin endometrium. Staining of the apical surface of the luminal epithelial cells (1) and of the glandular cells (2) with anti-Le $\mathrm{Y}^{Y}$ MAbs (a) and with Con A (b). Negative controls were performed without primary antibodies (c) and lectin Con $A(\mathbf{d})$ and with mouse $\operatorname{lgG}_{1}(\mathbf{e})$. Staining of positive controls: $\mathbf{f}$ - placental terminal villi with Con A, g - lung carcinoma tissues with the anti-Le ${ }^{Y}$ antibody. Magnification: $\times 400$

The importance of a sulfated residue (6-O-Su)GlcNAc for binding to the epitope recognized by MECA-79 MAbs has been especially noted [39].

Our findings on MECA-79 MAbs specificity demonstrate the high binding activity of these antibodies to type-2 glycan unit LacNAc. Glycan Galß1-4(6-O-Su)GlcNAc $\beta 1$-3GalNAc was not exposed on the microarray, but its sulfated form Gal $\beta 1-4(6-\mathrm{O}-\mathrm{Su}) \mathrm{GlcNAc} \beta$ (glycan № 203 in Table S1) was present, and we did not detect binding of MECA-79 to this glycan. Our data on the MECA-79 MAbs specificity do not contradict the literature, since our findings confirm the binding to LacNAc based glycans. However, (6-O-Su)GlcNAc $\beta$ residue is apparently not a unique factor for binding, but is recognized in complex with LacNAc, within a certain molecular recognition pattern of MECA-79 MAbs. Probably, a glycan epitope binding to MECA-79 is more complex than it has been shown before; this is quite explicable for MAbs obtained as a result of immunization with collagenase-dispersed BALB/c lymph node stroma, but not with one particular antigen.

MECA-79 is considered as one of the most relevant markers of endometrial receptivity [40]. A number of studies indicate that in a natural cycle, the expression of MECA-79 is significantly higher in fertile than in infertile patients [18]. Moreover, the absence of MECA-79 in the structures of endometrium in patients with infertility was shown to be associated with IVF failures [18]. Implantation and clinical pregnancy rates in patients with a higher MECA-79 expression were more than twice higher compared to patients with relatively low expression [41]. Our study allowed us to link lower MECA-79 expression with thin endometrium in patients with reproductive failures: embryo transfer in patients with endometrium less than $8 \mathrm{~mm}$ and decreased MECA-79 expression did not result in pregnancy, while $67 \%$ of patients with normal endometrium and higher MECA-79 expression succeeded to conceive. Therefore, assessment of MECA-79 expression may be used as a predictor of IVF failure and may help infertile patients with thin endometrium to avoid repeated useless treatment cycles.

The role of glycans in the pathophysiology of thin endometrium is also confirmed by the study of correlations between their expression levels in the main epithelial structures. The levels of expression of all studied glycans in two epithelial structures were comparable in patients of both groups, with a slight tendency towards higher expression in luminal epithelial cells. The luminal epithelium is the zone of primary contact with the trophectoderm, and the molecular "border" which the implanting embryo must overcome [13, 42]. The glands regulate uterine receptivity, luminal fluid homeostasis, blastocyst implantation, and embryo and placenta development at early stages $[29,36]$. Both glandular and luminal epithelium determine the receptivity of the endometrium but perform different functions: regulation of implantation and reception of the embryo, respectively. It is known that during endometrial adenogenesis, differentiation and budding of glandular epithelium from luminal epithelium occurs, followed by invagination and extensive tubular coiling and branching through uterine stroma into the myometrium [36, 43, 44] Having differentiated from the luminal epithelium, the glandular compartment apparently retains the quantitative and, in general, the qualitative composition of the glycocalyx.

Worth noting is that a correlation between the contents of fucosylated glycoconjugates with type-2 unit stained with UEA-I, was found in the glycocalyx of luminal and glandular epithelial cells both in thin and normal endometrium. It is known that UEA-I specifically stains antigen $\mathrm{H}$ type 2 [Fuc $\alpha 1-2 \mathrm{Gal} \beta 1-4 \mathrm{GlcNAc} \beta-\mathrm{R}$ ], 

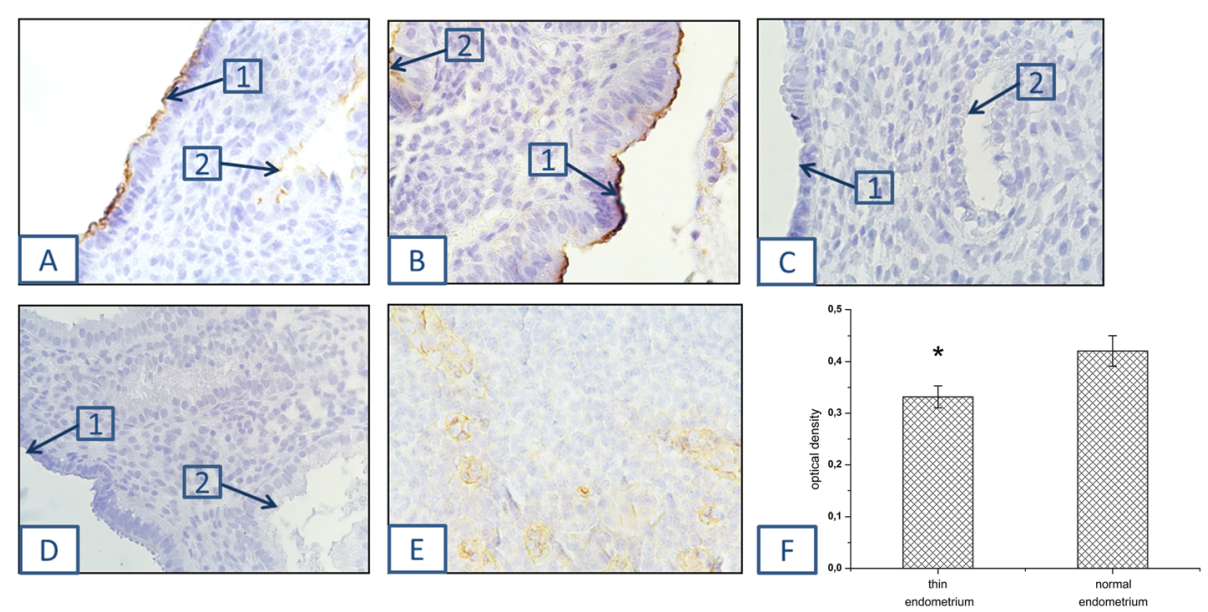

Fig. 2 Immunolocalization of carbohydrate epitopes MECA-79 on the apical surface of the luminal epithelial cells. a - thin and $\mathbf{b}$ - normal endometrium; (1) - luminal cells and (2) - glandular cells. Negative controls were performed without primary antibodies (c) and with isotypic rat IgM (d). Positive control - lymph nodes in breast cancer $(\mathbf{e})$. Magnification: $\times 400$. Histograms demonstrate relative expression of carbohydrate epitopes MECA-79 in thin $(n=14)$ and normal $(n=18)$ endometrium. The bar represents optical density as a mean value \pm standard deviation. ${ }^{*} p<0.05$ compared to normal endometrium (f)

one of the five types of carbohydrate cores for biosynthesis of antigens of the AB0 system and related antigens of the Lewis system, including $\mathrm{Le}^{\mathrm{Y}}[\mathrm{Fuc} \alpha 1-2 \mathrm{Gal} \beta 1$ 4(Fuc $\alpha 1-3)$ GlcNAc $\beta-R]$ [45]. Gal $\beta 1-4$ GlcNAc $\beta$ is a precursor of both fucosylated antigens and is specifically detected by ECL lectin. In both study groups, a significant correlation between the contents of LacNac residues in the superficial and glandular epithelium was detected only after pretreatment of endometrial tissue with neuraminidase followed by staining with ECL $(\mathrm{N}+\mathrm{ECL})$; this indicates the interrelated expression of not terminal, but subterminal $\mathrm{N}$-acetyllactosamine residues in both investigated structures. Noteworthy, in normal endometrium there is a tendency towards a higher expression of subterminal LacNAc residues in the surface epithelium, while in the thin endometrium, on the contrary, the expression of subterminal LacNAc residues it is higher in glandular epithelium. At the same time, the expression of subterminal $\mathrm{N}$-acetyllactosamine residues in the epithelial compartments of patients of both groups is higher, than of other glycans; this demonstrates wide representation of glycans with a type- 2 unit in the glycocalyx of the endometrial epithelial structures.

The terminal unsubstituted residues of LacNAc were found at the apical membranes of a few scattered glandular epithelial cells in normal endometrium, but could

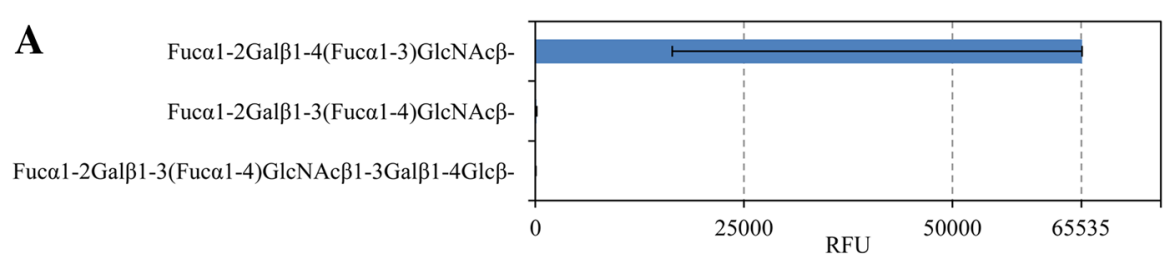

B

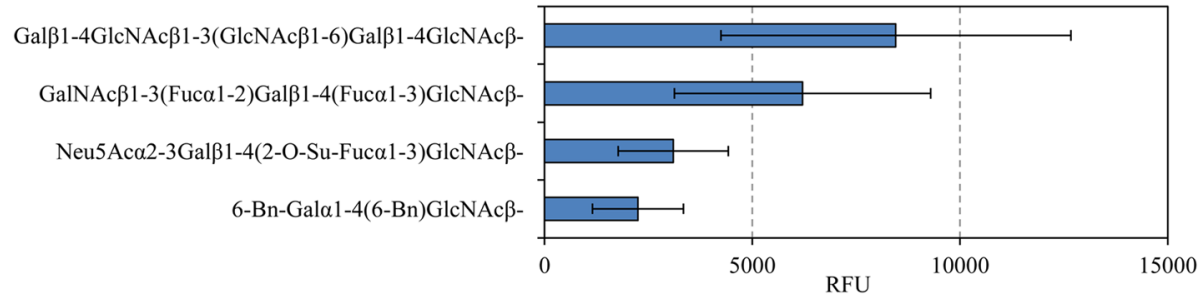

Fig. 3 Top ligands for anti-Le $e^{Y}$ MAbs (a) and MECA-79 MAbs (b). For structures and short names of all ligands of the microarray chip see

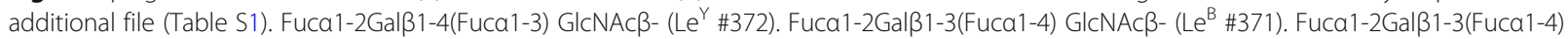

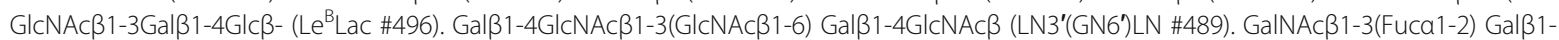

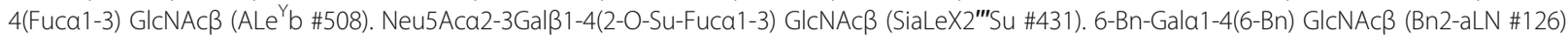


be seen more frequently in atrophic, weakly proliferating glandular epithelial cells [46]. We found no significant intergroup differences in the expression of both terminal and subterminal residues of $\mathrm{N}$-acetyllactosamine, as well as of fucosylated glycans stained with UEA-I; however, similar correlations of the expression of the corresponding glycoconjugates in the studied epithelial compartments of thin and normal endometrium apparently reflect the universal patterns of expression of glycans' functional residues, common for glycoconjugates of the endometrial glycocalyx, and may feature appropriate changes during the middle secretory phase, which do not affect endometrial receptivity.

In the study of $\alpha$ (1-2)-fucosylated glycans, special attention was paid to $\mathrm{Le}^{\mathrm{Y}}$ glycan, one of the key molecules mediating cell junctions, which is expressed in the secretory phase of the cycle as a part of the carbohydrate chains of $\alpha \mathrm{v} \beta 3$ integrin of the epithelial cells of the endometrium [47]. It was experimentally proved that $\mathrm{Le}^{\mathrm{Y}}$ can function as a regulatory and signaling molecule, since its inhibition is associated with impaired expression of matrix metalloproteinase 9 (MMP-9), epidermal growth factor (EGF), epidermal growth factor receptor (EGFR), and with the blockade of the DAG/PKC signaling pathway, which regulates cell growth, division, and differentiation [48]. It was found that the expression of $\mathrm{Le}^{\mathrm{Y}}$ and FUT4, an enzyme adding the Fuco1-3 residue to type-2 unit, correlates with a high reception of RL952 cells and activates the EGFR/MAPK signaling pathway [49]. Blocking of $\mathrm{Le}^{\mathrm{Y}}$ glycan biosynthesis in RL-95-2 endometrial cells line in vitro leads to a decrease in their adhesion to JAR embryonic cells and negatively regulates the $\alpha \mathrm{v} \beta 3 / \mathrm{FAK}$ signaling pathway which is responsible for invasion and migration of invading cells [50]. This confirms the key role of $\mathrm{Le}^{\mathrm{Y}}$ in intercellular contacts during implantation [45].

Our data showed that though overall $\mathrm{Le}^{\mathrm{Y}}$ expression levels in normal and thin endometrium were almost similar, there was a certain correlation between the $\mathrm{Le}^{\mathrm{Y}}$ levels in the two epithelial structures of thin endometrium, while in normal endometrium no correlation could be found. This phenomenon probably reflects the tissue homeostasis disorder, essential for morphogenesis of the endometrial functional layer and blastocyst acceptance, since $\mathrm{Le}^{\mathrm{Y}}$ plays a key role in intercellular contacts during implantation [45]. The patterns of specificity of anti-Le ${ }^{\mathrm{Y}}$ MAbs and UEA-I are similar, but not absolutely. As we have shown earlier, UEA-I has a broader specificity and recognizes fucosylated LacNAc structures, including $\mathrm{H}$ type 2 and $\mathrm{Le}^{\mathrm{Y}}$ antigens and some other LacNAc-containing glycans [51]. Apparently, thin endometrium has specific $\mathrm{Le}^{\mathrm{Y}}$ glycan expression which affects the endometrium receptivity, while expression of glycans containing type-2 unit is crucial for endometrial tissue, since LacNAc and its derivatives are the basis for biosynthesis of branched glycans abundantly present in the endometrium [52]. A pattern similar to $\mathrm{Le}^{\mathrm{Y}}$ was observed for mannose-rich glycans (staining with Con A) in the epithelial compartments of thin endometrium. A review of available literature provided evidence of an altered pattern of Con A binding in atrophic and hyperplastic postmenopausal endometrium [53], and in neoplastic endometrium with poor histological differentiation [54]. We have not found such correlations in normal endometrium. We assume that with the differentiation of luminal epithelium into the glandular epithelium and with the emergence of new functions in the normal endometrium with high receptivity, the correlation between the expression of certain glycans in the two epithelial structures vanish, reflecting adequate differentiation of epithelial cells. In thin endometrium the processes of differentiation of the glandular epithelium are probably impaired; this may negatively affect the maturity and functional activity of the cells.

\section{Conclusion}

Therefore, our preliminary study revealed specific expression of mannose-rich glycans and LacNAccontaining oligosaccharides (including $\mathrm{Le}^{\mathrm{Y}}$ and glycans containing LacNAc type- 2 unit, which have been identified as potential ligands to MECA-79 MAbs) in the epithelial compartments of thin endometrium involved in the interactions with the blastocyst during the implantation window. These changes may be essential for morphogenesis of the endometrial functional layer, resulting in the tissue deficiency and incapability to support the development of a haemochorial interface, and this, in turn, may affect the blastocyst implantation. We believe that alteration in the endometrial glycosylation pattern, namely the glycocalyx composition in the epithelial compartments, may contribute to the pathophysiology of thin endometrium and explain its low receptivity. Undoubtedly, a more profound study of a specific glycosylation pattern may provide insight into the pathophysiology of thin endometrium.

\footnotetext{
Abbreviations

AMH: Anti Mullerian hormone; ART: Assisted reproductive technologies; BMI: Body mass index; Con A: Concanavalin A from Canavalia ensiformis; EGF: Epidermal growth factor; EGFR: Epidermal growth factor receptor; ECL: Erythrina cristagalli lectin; ET: Embryo transfer; FSH: Follicle-stimulating hormone; FUT4: Fucosyltransferase 4; GlyCAM-1: Glycosylation-dependent cell adhesion molecule-1; GT: Gonadotropins; GnRH-ant: Gonadotropinreleasing hormone antagonists; IHC: Immunohistochemical;

ICSI: Intracytoplasmic sperm injection; IVF: In vitro fertilization; LacNAc: Nacetyllactosamine; LH: Luteinizing hormone; Le ${ }^{Y}$ : Lewis ${ }^{Y} ;$ MAL-II: Maackia amurensis lectin-II; MMP-9: Matrix metalloproteinase 9; MAbs: Monoclonal antibodies; MAdCAM-1: Mucosal addressin cell adhesion molecule-1; $\mathrm{N}$ : Neuraminidase; PNAd: Peripheral lymph node addressin; rFSH: Recombinant follicle-stimulating hormone; RFU: Relative fluorescence units; SNA: Sambucus nigra lectin; TVP: Transvaginal puncture; UEA-I: Ulex europaeus agglutinin-l; WL: Vicia villosa lectin
} 


\section{Supplementary Information}

The online version contains supplementary material available at https://doi. org/10.1186/s12958-021-00750-z.

Additional file 1: Table S1. The list of glycans. This Table represents all glycans (oligo- and polysaccharides) printed on microarrays used in this work

Additional file 2: Table S2. Glycoconjugates contents on the apical surface of the luminal and glandular epithelial cells in patients with "thin" endometrium (group 1) and patients with normal endometrium (group 2).

\section{Acknowledgments}

The authors would like to thank the staff of the 2nd Pathology Deprtment, National Medical Research Center for Obstetrics, Gynecology, and

Perinatology named after Academician V.I. Kulakov of the Ministry of Health of the Russian Federation, Moscow, Russia for their assistance. The authors are grateful to Nadezhda Shilova from the Laboratory of carbohydrates of the M.M. Shemyakin-Yu.A. Ovchinnikov Institute of Bioorganic Chemistry of the Russian Academy of Science, Moscow, Russia, for discussion and comments.

\section{Authors' contributions}

M.M.Z, N.V.D., and G.T.S. designed the study, acquired and interpreted data and wrote the manuscript. G.V.K., N.M.F., N.R.K., N.F.A., A.V.A., A.A.D, and A.I.S performed experiments, assisted in acquiring and interpreting data. E.L.Y. designed the study and wrote the manuscript. All authors read and approved the final manuscript.

\section{Funding}

The study was performed as a part of the State assignment (State Registration No.AAAA-A18-118053190022-8).

\section{Availability of data and materials}

The datasets used and/or analyzed during the current study are available from the corresponding author on reasonable request.

\section{Declarations}

Ethics approval and consent to participate

The study was approved by the IRB of the National Medical Research Center for Obstetrics, Gynecology, and Perinatology named after Academician V.I. Kulakov, Moscow, Russia (PR№ 10-20/10/2016).

\section{Consent for publication}

All women gave written informed consent to participate in the study.

\section{Competing interests}

The authors declare no competing interests.

\section{Author details}

'Laboratory of Clinical Immunology, National Medical Research Center for Obstetrics, Gynecology, and Perinatology named after Academician V.I. Kulakov of the Ministry of Healthcare of Russian Federation, Oparina str. 4 Moscow, Russian Federation 117997. ${ }^{2}$ R\&D Department, National Medical Research Center for Obstetrics, Gynecology, and Perinatology of Ministry of Healthcare of Russian Federation, Oparina str. 4, Moscow 117997, Russia. ${ }^{3}$ First Moscow State Medical University named after I.M. Sechenov, Trubetskaya str. 8-2, Moscow 119991, Russia. ${ }^{4}$ Department of Pathology, National Medical Research Center for Obstetrics, Gynecology, and Perinatology of Ministry of Healthcare of Russian Federation, Oparina str. 4 Moscow 117997, Russia. ${ }^{5}$ Department of International Cooperation, National Medical Research Center for Obstetrics, Gynecology, and Perinatology of Ministry of Healthcare of Russian Federation, Oparina str. 4, Moscow 117997, Russia. ${ }^{6}$ Department of Assisted Technologies in Treatment of Infertility, National Medical Research Center for Obstetrics, Gynecology, and Perinatology of Ministry of Healthcare of Russian Federation, Oparina str. 4, Moscow 117997, Russia.
Received: 3 August 2020 Accepted: 26 April 2021

Published online: 15 May 2021

\section{References}

1. Gonen Y, Casper RF, Jacobson W, Blankier J. Endometrial thickness and growth during ovarian stimulation: a possible predictor of implantation in in vitro fertilization. Fertil Steril. 1989:52(3):46-50.

2. Zhang T, Li Z, Ren X, et al. Endometrial thickness as a predictor of the reproductive outcomes in fresh and frozen embryo transfer cycles: a retrospective cohort study of 1512 IVF cycles with morphologically goodquality blastocyst. Medicine. 2018;97(4):1-9.

3. Liu KE, Hartman M, Hartman A, et al. The impact of a thin endometrial lining on fresh and frozen-thaw IVF outcomes: an analysis of over 40000 embryo transfers. Hum Reprod. 2018;33(10):1883-8.

4. Oliveira JB, Baruffi RL, Mauri AL, et al. Endometrial ultrasonography as a predictor of pregnancy in an in-vitro fertilization programme after ovarian stimulation and gonadotrophin-releasing hormone and gonadotrophins. Hum Reprod. 1997:12(11):2515-8.

5. Mahajan N, Sharma S. The endometrium in assisted reproductive technology: how thin is thin? J Hum Reprod Sci. 2016;9(1):3-8.

6. Dix $E$, Check JH. Successful pregnancies following embryo transfer despite very thin late proliferative endometrium. Clin Exp Obstet Gynecol. 2010; 37(1):15-6.

7. von Wolff $M$, Fäh $M$, Roumet $M$, et al. Thin endometrium is also associated with lower clinical pregnancy rate in unstimulated menstrual cycles: a study based on natural cycle IVF. Front Endocrinol. 2018;9:776.

8. Oron G, Hiersch L, Rona S, et al. Endometrial thickness of less than $7.5 \mathrm{~mm}$ is associated with obstetric complications in fresh IVF cycles: a retrospective cohort study. Reprod BioMed Online. 2018;37(3):341-8.

9. Revel A. Defective endometrial receptivity. Fertil Steril. 2012;97(5):1028-32.

10. Mouhayar $\mathrm{Y}$, Sharara FI. Modern management of thin lining. Middle East Fertil Soc J. 2017;22(1):1-12.

11. Alfer J, Happel L, Dittrich $R$, et al. Insufficient angiogenesis: cause of abnormally thin endometrium in subfertile patients? Geburtshilfe Frauenheilkd. 2017:77(7):756-64.

12. Carson DD. The glycobiology of implantation. Front Biosci. 2002;7:d1535-44.

13. Aplin JD, Jones CJ. Fucose, placental evolution and the glycocode. Glycobiology. 2012;22(4):470-8

14. Clark GF. Functional glycosylation in the human and mammalian uterus. Fertil Res Pract. 2015;1:1-12.

15. Aplin JD. Glycans as biochemical markers of human endometrial secretory differentiation. J Reprod Fertil. 1991;92(2):525-41.

16. Gu J, Isaji T, Xu Q, et al. Potential roles of $\mathrm{N}$-glycosylation in cell adhesion. Glycoconj J. 2012;29(8-9):599-607.

17. Nejatbakhsh R, Kabir-Salmani M, Dimitriadis E, et al. Subcellular localization of L-selectin ligand in the endometrium implies a novel function for pinopodes in endometrial receptivity. Reprod Biol Endocrinol. 2012:10:46.

18. Margarit L, Gonzalez D, Lewis PD, et al. L-Selectin ligands in human endometrium: comparison of fertile and infertile subjects. Hum Reprod. 2009;24(11):2767-77

19. Miller DL, Jones CJP, Aplin JD, Nardo LG. Altered glycosylation in periimplantation phase endometrium in women with stages III and IV endometriosis. Hum Reprod. 2010;25(2):406-11.

20. Noyes W, Hertig Al, Rock J. Dating the endometrial biopsy. Fertil Steril. 1950; $1 \cdot 3-25$

21. Abdurakhmanova NF, Gvozdeva AD, Ziganshina MM, Dolgushina NV. The results of assisted reproductive technology programs in patients with "thin" endometrium. Gynecology (Rus). 2019;21(1):23-7.

22. Gardner DK, Schoolcraft WB. Culture and transfer of human blastocysts. Curr Opin Obstet Gynecol. 1999;11(3):307-11.

23. Yeh J-C, Hiraoka N, Petryniak B, et al. Novel sulfated lymphocyte homing receptors and their control by a core1 extension $31,3-\mathrm{N}-$ Acetylglucosaminyltransferase. Cell. 2001;105(7):957-69.

24. Jones CJP, Twink Allen WR, Wilsher S. A lectin histochemical study to detect variation in glycosylation at the feto-maternal interface in three interbreeding equine species. Placenta. 2017:58:115-21.

25. Jones CJP, Denton J, Fazleabas AT. Morphological and glycosylation changes associated with the endometrium and ectopic lesions in a baboon model of endometriosis. Hum Reprod. 2006;21(12):3068-80. 
26. Blixt $\mathrm{O}$, Head $\mathrm{S}$, Mondala $\mathrm{T}$, et al. Printed covalent glycan array for ligand profiling of diverse glycan binding proteins. Proc Natl Acad Sci. 2004; 101(49):17033-8

27. Miravet-Valenciano JA, Rincon-Bertolin A, Vilella F, Simon C. Understanding and improving endometrial receptivity. Curr Opin Obstet Gynecol. 2015; 27(3):187-92.

28. Aoki D, Kawakami H, Nozawa S, et al. Differences in lectin binding patterns of normal human endometrium between proliferative and secretory phases. Histochemistry. 1989;92(3):177-84.

29. Kimber SJ. Molecular interactions at the maternal-embryonic interface during the early phase of implantation. Semin Reprod Med. 2000;18(3):237-53.

30. Aplin JD, Ruane PT. Embryo-epithelium interactions during implantation at a glance. J Cell Sci. 2017;130(1):15-22

31. Yu M, Qin H, Wang H, Liu J, Liu S, Yan Q. N-glycosylation of uterine endometrium determines its receptivity. J Cell Physiol. 2020;235(2):1076-89.

32. Yan D, Lin X. Shaping morphogen gradients by proteoglycans. Cold Spring Harb Perspect Biol. 2009;1(3):a002493.

33. Podbilewicz B. Sweet control of cell migration, cytokinesis and organogenesis. Nat Cell Biol. 2004;6(1):9-11.

34. Toole BP. Hyaluronan in morphogenesis. Semin Cell Dev Biol. 2001;12(2):79-87.

35. Bhat $\mathrm{R}$, Belardi $\mathrm{B}$, Mori $\mathrm{H}$, et al. Nuclear repartitioning of galectin-1 by an extracellular glycan switch regulates mammary morphogenesis. Proc Natl Acad Sci. 2016;113(33):E4820-7.

36. Kelleher AM, Burns GW, Behura $\mathrm{S}$, et al. Uterine glands impact uterine receptivity, luminal fluid homeostasis and blastocyst implantation. Sci Rep. 2016;6(1):38078

37. Hemmerich S, Butcher EC, Rosen SD. Sulfation-dependent recognition of high endothelial venules (HEV)-ligands by L-selectin and MECA 79, and adhesion-blocking monoclonal antibody. J Exp Med. 1994;180(6):2219-26.

38. Hemmerich S, Rosen SD. 6'-sulfated sialyl Lewis $\mathrm{x}$ is a major capping group of GlyCAM-1. Biochemistry. 1994;33(16):4830-5.

39. Hoshino $\mathrm{H}$, Ohta $\mathrm{M}$, Ito $\mathrm{M}$, et al. Apical membrane expression of distinct sulfated glycans represents a novel marker of cholangiolocellular carcinoma. Lab Investig. 2016;96(12):1246-55.

40. Craciunas L, Gallos I, Chu J, et al. Conventional and modern markers of endometrial receptivity: a systematic review and meta-analysis. Hum Reprod Update. 2019;25(2):202-23.

41. Foulk RA, Zdravkovic T, Genbacev O, Prakobphol A. Expression of L-selectin ligand MECA-79 as a predictive marker of human uterine receptivity. J Assist Reprod Genet. 2007;24(7):316-21.

42. Wang B, Sheng J-Z, He R-H, et al. High expression of L-selectin ligand in secretory endometrium is associated with better endometrial receptivity and facilitates embryo implantation in human being. Am J Reprod Immunol. 2008;60(2):127-34.

43. Gray CA, Bartol FF, Tarleton BJ, et al. Developmental biology of uterine glands. Biol Reprod. 2001;65(5):1311-23.

44. Kelleher AM, DeMayo FJ, Spencer TE. Uterine glands: developmental biology and functional roles in pregnancy. Endocr Rev. 2019;40(5):1424-45.

45. Gu J, Sui L-L, Cui D, et al. Effects of LeY glycan expression on embryo implantation. Eur Rev Med Pharmacol Sci. 2016;20(16):33-5.

46. Ravn V, Mandel U, Svenstrup B, Dabelsteen E. N-acetyllactosamine and sialosyl-N-acetyllactosamine in normal and malignant human endometrium. Glycosylation Dis. 1994;1:271-8.

47. Lessey BA. Adhesion molecules and implantation. J Reprod Immunol. 2002; 55(1-2):101-12.

48. Li Y, Ma K, Sun P, et al. LeY oligosaccharide upregulates DAG/PKC signaling pathway in the human endometrial cells. Mol Cell Biochem. 2009;331(1-2):1-7.

49. Liu S, Yang $X$, Wang J, et al. Differential expression of LeY and fucosyltransferase IV correlates with the receptivity of RL95-2 and HEC-1A human uterine epithelial cells. Cell Biol Int. 2012;36(5):469-74.

50. Zhang $\mathrm{D}$, Wei J, Wang J, et al. Difucosylated oligosaccharide Lewis $Y$ is contained within integrin av 33 on RL95-2 cells and required for endometrial receptivity. Fertil Steril. 2011;95(4):1446-1451.e1.

51. Ziganshina MM, Kulikova GV, Fayzullina NM, et al. Expression of fucosylated glycans in endothelial glycocalyces of placental villi at early and late fetal growth restriction. Placenta. 2020;90:98-102.

52. Kupper CE, Rosencrantz RR, Henßen B, et al. Chemo-enzymatic modification of poly-N-acetyllactosamine (LacNAc) oligomers and N,Ndiacetyllactosamine (LacDiNAc) based on galactose oxidase treatment. Beilstein J Org Chem. 2012;8:712-25.
53. Noci I, Gheri G, Bryk SG, et al. Aging of the human endometrium: periimplantation phase endometrium does not show any age-dependent variation in lectin binding. Eur J Obstet Gynecol Reprod Biol. 1996;64(1): $11-21$.

54. Sivridis E, Giatromanolaki A, Koukourakis M, Agnantis N. Lectin-binding patterns in normal, hyperplastic and neoplastic endometrium: the prognostic value of concanavalin a. Virchows Arch. 2000;436(1):52-8.

\section{Publisher's Note}

Springer Nature remains neutral with regard to jurisdictional claims in published maps and institutional affiliations.
Ready to submit your research? Choose BMC and benefit from:

- fast, convenient online submission

- thorough peer review by experienced researchers in your field

- rapid publication on acceptance

- support for research data, including large and complex data types

- gold Open Access which fosters wider collaboration and increased citations

- maximum visibility for your research: over $100 \mathrm{M}$ website views per year

At $\mathrm{BMC}$, research is always in progress.

Learn more biomedcentral.com/submissions 